This is an electronic reprint of the original article. This reprint may differ from the original in pagination and typographic detail.

Author(s): Prassa, Vaia; Nikšic, T.; Vretenar, D.

Title: $\quad$ Structure of transactinide nuclei with relativistic energy density functionals

Year: $\quad 2013$

Version:

Please cite the original version:

Prassa, V., Nikšic, T., \& Vretenar, D. (2013). Structure of transactinide nuclei with relativistic energy density functionals. Physical Review C, 88(4), Article 044324. https://doi.org/10.1103/PhysRevC.88.044324

All material supplied via JYX is protected by copyright and other intellectual property rights, and duplication or sale of all or part of any of the repository collections is not permitted, except that material may be duplicated by you for your research use or educational purposes in electronic or print form. You must obtain permission for any other use. Electronic or print copies may not be offered, whether for sale or otherwise to anyone who is not an authorised user. 


\title{
Structure of transactinide nuclei with relativistic energy density functionals
}

\author{
V. Prassa, ${ }^{1,2}$ T. Nikšić, ${ }^{2}$ and D. Vretenar ${ }^{2}$ \\ ${ }^{1}$ Physics Department, University of Jyväskylä, P.O. Box 35 (YFL) FI-40014, Finland \\ ${ }^{2}$ Physics Department, Faculty of science, University of Zagreb, 10000 Zagreb, Croatia
}

(Received 9 August 2013; revised manuscript received 23 September 2013; published 21 October 2013)

\begin{abstract}
A microscopic theoretical framework based on relativistic energy density functionals (REDFs) is applied to studies of shape evolution, excitation spectra, and decay properties of transactinide nuclei. Axially symmetric and triaxial relativistic Hartree-Bogoliubov (RHB) calculations, based on the functional DD-PC1 and with a separable pairing interaction, are performed for the even-even isotopic chains between Fm and Fl. The occurrence of a deformed shell gap at neutron number $N=162$ and its role on the stability of nuclei in the region around $Z=108$ is investigated. A quadrupole collective Hamiltonian, with parameters determined by self-consistent constrained triaxial RHB calculations, is used to examine low-energy spectra of No, Rf, Sg, Hs, and Ds with neutron number in the interval $158 \leqslant N \leqslant 170$. In particular, we analyze the isotopic dependence of several observables that characterize the transitions between axially symmetric rotors, $\gamma$-soft rotors, and spherical vibrators. An interesting example of a possible occurrence of shape-phase transitions and critical-point phenomena in this mass region is explored.
\end{abstract}

DOI: 10.1103/PhysRevC.88.044324

PACS number(s): 21.60.Jz, 21.10.Dr, 21.10.Re, 27.90.+b

\section{INTRODUCTION}

Enormous progress has been achieved in recent years in the synthesis and structure studies of superheavy elements [1-6]. These experiments present one of the most active fields at the forefront of nuclear physics research. While available data on the heaviest elements are often restricted just to the existence of a particular nuclide and its main decay modes, detailed spectroscopic properties have been investigated, both experimentally and theoretically, for heavy actinides and transactinide nuclei [7-9]. Two complementary classes of theoretical methods are used in nuclear structure studies in this mass region: the traditional macroscopic-microscopic approach [10-13], and the framework of microscopic self-consistent mean-field models, based on realistic effective internucleon interactions or energy density functionals [14-16]. The latter have not yet achieved the accuracy of the former in the description of the evolution of shell structure, deformed gaps, $K$ isomers, $\alpha$-decay energies and lifetimes, and fission barriers $[13,15]$. This is mostly because microscopic self-consistent meanfield models employ global effective interactions or density functionals that are used for the entire chart of nuclides, rather than potentials parametrized specifically to the mass region of interest. Nevertheless, a vast body of high-quality microscopic self-consistent mean-field calculations of the structure of very heavy and superheavy nuclei has been reported in the last fifteen years or so (for an exhaustive list of references, we refer the reader to $[13,15,17])$. In a large number of cases excellent agreement with data and unique predictions have been obtained.

The purpose of this work is to perform a microscopic self-consistent mean-field study of the transactinides, from Fm and Fl, with neutron number in the interval $154 \leqslant N \leqslant 172$. Except for a few two-neutron separation energies and slightly more data on $\alpha$-decay energies, spectroscopic data on these nuclei are not available yet. For instance, the heaviest nucleus for which the rotational band structure has been observed to date is ${ }^{256} \mathrm{Rf}$ [18] (up to spin 20ћ), and the experimental kinematic and dynamic moments of inertia have accurately been reproduced using a cranked shell model with pairing correlations treated by a particle-number conserving method [19], and the cranked relativistic Hartree-Bogoliubov model [20]. Even though spectroscopic properties of the nuclides considered in this study might not be experimentally accessible in the near future, it is nevertheless interesting to analyze microscopic predictions for the occurrence of deformed gaps, two-neutron separation energies, $Q_{\alpha}$ values, evolution of shapes and characteristics of low-energy collective spectra of these systems.

In Sec. II we briefly outline the theoretical framework of deformation-constrained self-consistent mean-field methods and the corresponding collective Hamiltonian, based on relativistic energy density functionals. The occurrence of the $N=162$ deformed neutron gap is analyzed in Sec. III, and the evolution of quadrupole shapes and low-energy collective spectra are discussed in Sec. IV. Section V contains a brief summary and an outlook for future studies.

\section{THEORETICAL FRAMEWORK}

The tool of choice for theoretical studies of the structure of medium-heavy and heavy nuclei is the framework of energy density functionals (EDFs) $[14,16]$. Self-consistent mean-field models based on semi-empirical nuclear energy density functionals provide an accurate and reliable microscopic description of nuclear structure phenomena over the whole nuclide chart. EDF-based structure models have also been developed that go beyond the static mean-field approximation, and include collective correlations related to the restoration of broken symmetries and to fluctuations of collective variables. These models are employed in analyses of structure phenomena related to shell evolution, including detailed predictions of excitation spectra and electromagnetic transition rates. Relativistic EDF-based methods [21-23], in particular, have successfully been applied to nuclear structure 
studies in different mass regions including superheavy nuclei, and have reached the level of accuracy of their nonrelativistic counterparts, e.g., models derived from Skyrme functionals.

For a microscopic self-consistent study of the structure of transactinide nuclei, in this work we employ the relativistic functional DD-PC1 [24]. Starting from microscopic nucleon self-energies in nuclear matter, and empirical global properties of the nuclear matter equation of state, the coupling parameters of DD-PC1 were fine-tuned to the experimental masses of a set of 64 deformed nuclei in the mass regions $A \approx 150-180$ and $A \approx 230-250$. The functional has been further tested in calculations of ground-state properties of medium-heavy and heavy nuclei, including binding energies, charge radii, deformation parameters, neutron skin thickness, and excitation energies of giant multipole resonances. It has also successfully been applied in a number of studies based on the quadrupole collective Hamiltonian, that have analyzed the evolution of shell structure and low-energy collective spectra in the $N=28$ isotones [25], medium-heavy and heavy nuclei [23,26] and, very recently, in superheavy systems [17,27,28].

For a quantitative description of open-shell nuclei it is necessary to consider also pairing correlations. The relativistic Hartree-Bogoliubov (RHB) framework [21,22] provides a unified description of particle-hole $(p h)$ and particle-particle $(p p)$ correlations on a mean-field level by combining two average potentials: the self-consistent mean field that encloses all the long range $p h$ correlations, and a pairing field $\hat{\Delta}$ which sums up the $p p$ correlations. In this study we will carry out axially symmetric and triaxial RHB calculations with the $p h$ effective interaction determined by the DD-PC1 functional. A pairing force separable in momentum space: $\left\langle k\left|V^{1} S_{0}\right| k^{\prime}\right\rangle=-G p(k) p\left(k^{\prime}\right)$ will be used in the $p p$ channel $[29,30]$. By assuming a simple Gaussian ansatz $p(k)=e^{-a^{2} k^{2}}$, the two parameters $G$ and $a$ were adjusted to reproduce the density dependence of the gap at the Fermi surface in nuclear matter, calculated with a Gogny force. For the D1S parametrization [31] of the Gogny force the following values were determined: $G=-728 \mathrm{MeV} \mathrm{fm}^{3}$ and $a=0.644 \mathrm{fm}$ [29]. When transformed from momentum to coordinate space, the interaction takes the form:

$$
V\left(\boldsymbol{r}_{1}, \boldsymbol{r}_{2}, \boldsymbol{r}_{1}^{\prime}, \boldsymbol{r}_{2}^{\prime}\right)=G \delta\left(\boldsymbol{R}-\boldsymbol{R}^{\prime}\right) P(\boldsymbol{r}) P\left(\boldsymbol{r}^{\prime}\right) \frac{1}{2}\left(1-P^{\sigma}\right),
$$

where $\boldsymbol{R}=\frac{1}{2}\left(\boldsymbol{r}_{1}+\boldsymbol{r}_{2}\right)$ and $\boldsymbol{r}=\boldsymbol{r}_{1}-\boldsymbol{r}_{2}$ denote the centerof-mass and the relative coordinates, and $P(\boldsymbol{r})$ is the Fourier transform of $p(k): P(\boldsymbol{r})=1 /\left(4 \pi a^{2}\right)^{3 / 2} e^{-\boldsymbol{r}^{2} / 4 a^{2}}$.

To describe nuclei with general quadrupole shapes, the Dirac-Hartree-Bogoliubov equations are solved by expanding the nucleon spinors in the basis of a 3D harmonic oscillator in Cartesian coordinates. In the present study of transactinide nuclei calculations have been performed in a basis with $N_{f}^{\max }=16$ oscillator shells for the upper component, and $N_{f}^{\max }=17$ shells for the lower component of the Dirac spinors [32]. The map of the energy surface as a function of the quadrupole deformation is obtained by imposing constraints on the axial and triaxial quadrupole moments. The method of quadratic constraint uses an unrestricted variation of the function

$$
\langle\hat{H}\rangle+\sum_{\mu=0,2} C_{2 \mu}\left(\left\langle\hat{Q}_{2 \mu}\right\rangle-q_{2 \mu}\right)^{2},
$$

where $\langle\hat{H}\rangle$ is the total energy, and $\left\langle\hat{Q}_{2 \mu}\right\rangle$ denotes the expectation value of the mass quadrupole operators:

$$
\hat{Q}_{20}=2 z^{2}-x^{2}-y^{2} \text { and } \hat{Q}_{22}=x^{2}-y^{2} .
$$

$q_{2 \mu}$ is the constrained value of the multipole moment, and $C_{2 \mu}$ the corresponding stiffness constant.

The self-consistent solutions of the constrained triaxial RHB equations, that is, the single-quasiparticle energies and wave functions for the entire energy surface as functions of the quadrupole deformation, provide the microscopic input for the parameters of a collective Hamiltonian for vibrational and rotational degrees of freedom [33]. The five quadrupole collective coordinates are parametrized in terms of the two deformation parameters $\beta$ and $\gamma$, and three Euler angles $(\phi, \theta, \psi) \equiv \Omega$, which define the orientation of the intrinsic principal axes in the laboratory frame. The dynamics of the collective Hamiltonian is governed by the seven functions of the intrinsic deformations $\beta$ and $\gamma$ : the collective potential, the three mass parameters, and the three moments of inertia $\mathcal{I}_{k}$. These functions are determined by the microscopic nuclear energy density functional and the effective interaction in the $p p$ channel. The moments of inertia and the mass parameters are calculated in the cranking approximation, and the collective potential is obtained by subtracting the zero-point energy corrections from the total energy that corresponds to the solution of constrained RHB equations.

The collective Hamiltonian describes quadrupole vibrations, rotations, and the coupling of these collective modes. The corresponding eigenvalue problem is solved using an expansion of eigenfunctions in terms of a complete set of basis functions that depend on the deformation variables $\beta$ and $\gamma$, and the Euler angles $\phi, \theta$, and $\psi$ [33]. The diagonalization of the Hamiltonian yields the excitation energies and collective wave functions, that can be used to calculate expectation values of various observables and transition probabilities.

\section{THE $N=162$ NEUTRON GAP}

Ground-state observables often display characteristic signatures of the evolution of underlying single-nucleon shell structure. In Fig. 1 we plot the two-neutron separation energies of transactinide nuclei as functions of the neutron number. The theoretical values are obtained in the self-consistent relativistic Hartree-Bogoliubov formalism, using the functional DD-PC1 in the particle-hole channel and the separable pairing force Eq. (1), as described in the previous section. The separation energies $S_{2 n}$ correspond to axially symmetric equilibrium minima. A common feature is the decrease of $S_{2 n}$ with increasing neutron number. However, a more pronounced reduction is found between $N=162$ and $N=164$ for several isotopic chains, most notably $\mathrm{Rf}, \mathrm{Sg}$, and Hs. A sudden drop in separation energies with nucleon number is associated with pronounced shell gaps, and this effect is especially marked at spherical magic nucleon numbers. The effect shown in 


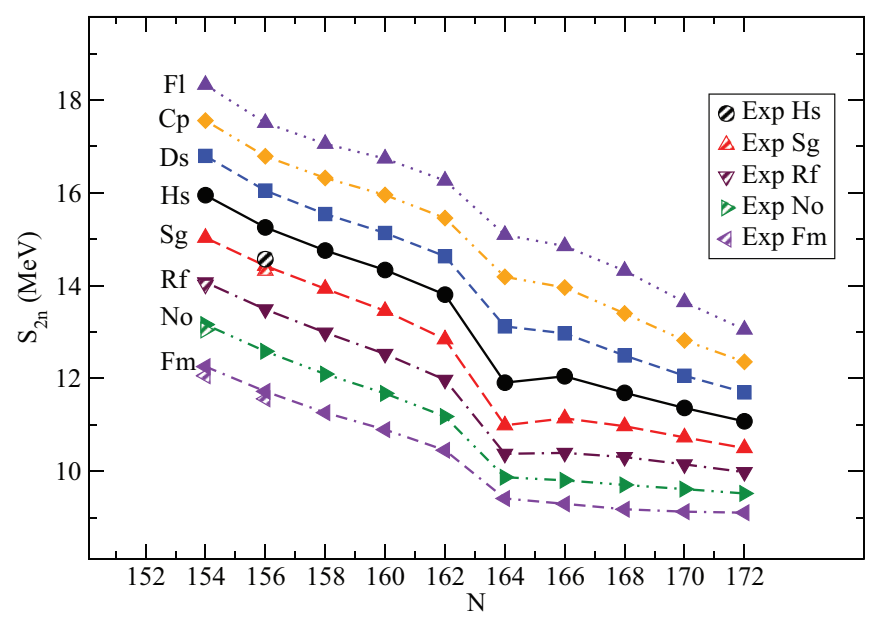

FIG. 1. (Color online) Two-neutron separation energies of transactinide nuclei as functions of the neutron number. Calculated values are denoted by filled symbols, available data by striped ones. Lines connect isotopes, the experimental values are from Ref. [37].

Fig. 1 indicates the occurrence of a neutron shell gap in deformed transactinide nuclei at $N=162$, in agreement with results of previous studies performed using the macroscopicmicroscopic approach $[13,34,35]$. Very similar results for the $S_{2 n}$ values and, in particular for the discontinuity at $N=162$, have recently been obtained in the self-consistent HartreeFock-Bogoliubov (HFB) calculation with the finite-range Gogny force with D1S [36]. We have also verified the convergence of the calculated separation energies with respect to the dimension of the 3D harmonic oscillator basis. The difference between separation energies calculated with $N_{f}^{\max }=16$ and $N_{f}^{\max }=18$ oscillator shells does not exceed $100 \mathrm{keV}$.

Very few experimental results are available for $S_{2 n}$ in this region of relatively neutron-rich transactinides [37], and the present calculation reproduces the data (cf. Fig. 1). To test the agreement of our self-consistent approach with experiment in more detail, in Fig. 2 we compare the predicted $\alpha$-decay

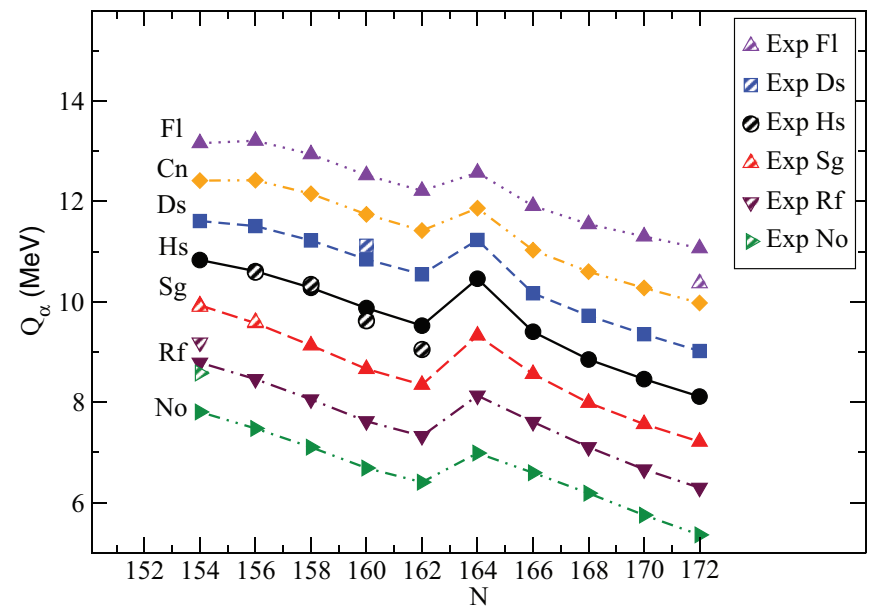

FIG. 2. (Color online) $Q_{\alpha}$ values for transactinide nuclei: lines connect theoretical values that correspond to isotopic chains, plotted in comparison to available data (striped symbols) from [37]. energies $Q_{\alpha}$, that is, energies of $\alpha$ particles emitted by even-even isotopes to available data [37]. These observables are measured independently of the corresponding masses and, therefore, can be used as an additional test of the accuracy of a given theoretical method. In the present calculation, assuming axial symmetry, the level of agreement is similar to that obtained in the HFB calculation with the Gogny force [36]. For $\alpha$-decay energies the subshell closure at $N=162$ manifests itself by the increase of $Q_{\alpha}$ values between $N=162$ and $N=164$.

Figure 3 displays the energies of canonical single-particle RHB neutron (left panel) and proton (right panel) states of ${ }^{270} \mathrm{Hs}$ as functions of the axial deformation parameter $\beta_{2}$. Continuous and dashed curves represent positive- and negative-parity levels, respectively. This nucleus is central to the region of transactinides considered in the present study, and its single-particle spectrum has been analyzed in a number of studies of the deformed $N=162$ shell gap $[13,35,36]$. In the present calculation with the DD-PC1 density functional, this gap is clearly identified in the Nilsson diagram of neutron single-particle levels at prolate deformation $\beta_{2} \approx 0.25$. As no particle number projection is performed, a pairing collapse occurs at the equilibrium deformation characterized by the large gap between neutron levels. In this case the position of the Fermi level is conventionally defined as the average energy between the last occupied and first unoccupied neutron levels. The size of the gap $2.17 \mathrm{MeV}$ at equilibrium deformation is, of course, much smaller than typical shell gaps in spherical magic nuclei but, nevertheless, it gives rise to the characteristic features of neutron separation energies and $Q_{\alpha}$ values shown in Figs. 1 and 2, respectively. The corresponding proton gap at equilibrium deformation (right panel of Fig. 3) is much less pronounced, that is, the size of the gap is only slightly larger than $1 \mathrm{MeV}$.

The central position of ${ }^{270} \mathrm{Hs}$ in this region of transactinides, that is, its role as a "deformed magic" nucleus, is also illustrated by the evolution of the deformed neutron shell gap of the $N=162$ isotones as a function of proton number, and the proton shell gap of $\mathrm{Hs}$ isotopes as a function of the number of neutrons, shown in Fig. 4. The former displays a pronounced peak at $Z=108$, and the proton gaps are largest for $N=162$ and $N=164$.

Ground-state properties and the evolution of single-nucleon shell structures, calculated assuming axially symmetric quadrupole shapes, already indicate the presence of interesting structural changes at and beyond $Z=108$ and $N=162$. Therefore, in the following section we will explore the triaxial shapes and collective excitation spectra of these heavy systems in a calculation that includes constrained self-consistent RHB deformation energy maps and the corresponding solutions of a quadrupole collective Hamiltonian for vibrational and rotational degrees of freedom.

\section{EVOLUTION OF SHAPES AND LOW-ENERGY COLLECTIVE SPECTRA}

The complex evolution of single-nucleon orbitals as functions of nuclear deformation and of nucleon number, 


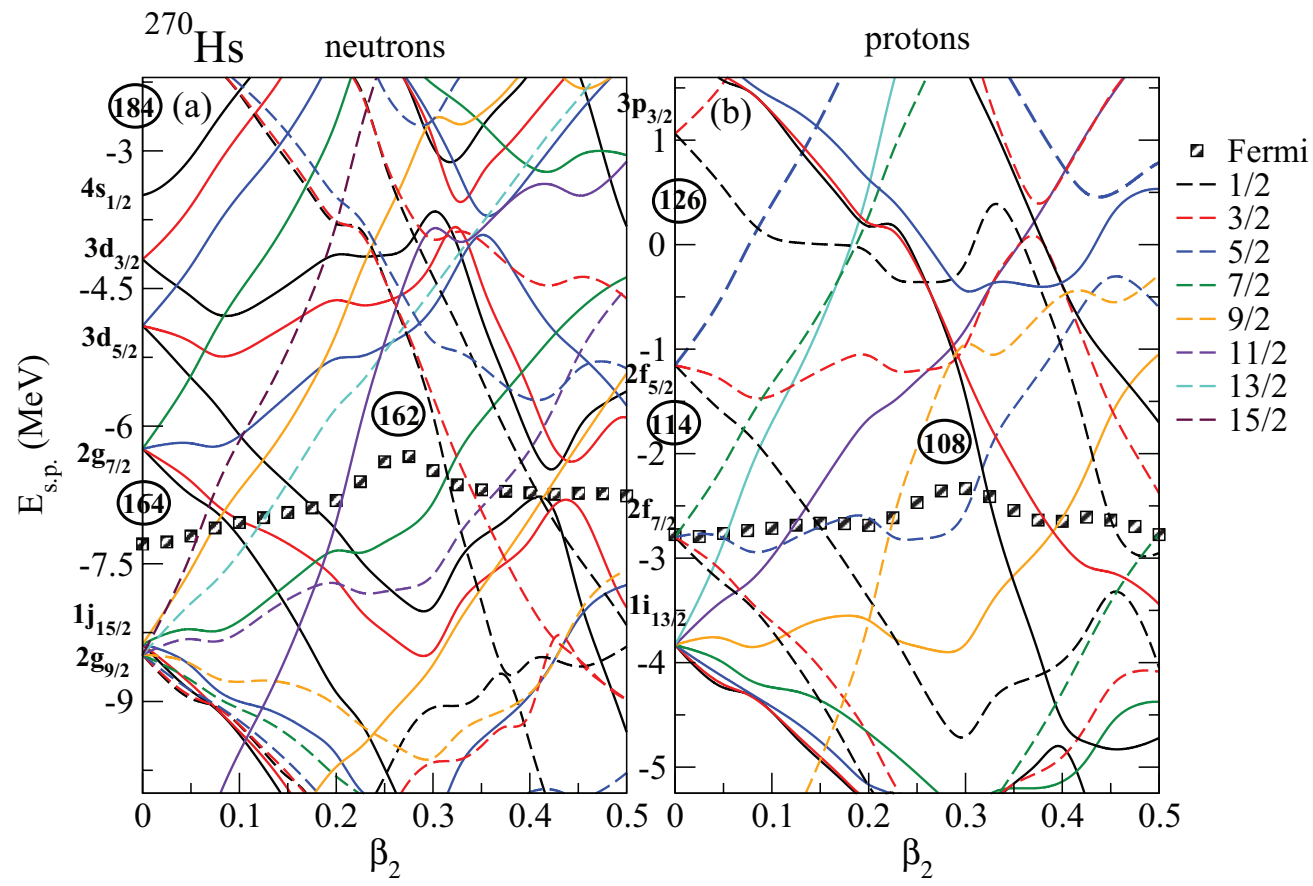

FIG. 3. (Color online) Neutron (left panel) and proton (right panel) single-particle energies of ${ }^{270} \mathrm{Hs}$ as functions of the axial deformation parameter $\beta_{2}$. Continuous and dashed curves represent positive- and negative-parity levels, respectively. The position of the Fermi level is denoted by the square symbols.

determines the transitions in equilibrium shapes. For the isotopic chains of No, $\mathrm{Rf}, \mathrm{Sg}, \mathrm{Hs}$, and Ds, this is illustrated in Figs. 5, 6, 7, 8, and 9, respectively, where we plot the results of constrained self-consistent triaxial RHB calculations of energy surfaces in the $\beta-\gamma$ plane $\left(0 \leqslant \gamma \leqslant 60^{\circ}\right)$. For each nucleus energies are normalized with respect to the binding energy of the equilibrium deformation, and the color code refers to the energy at each point on the surface relative to the minimum. To analyze the structure of nuclei in the vicinity of the gap at $N=162$, for each isotopic chain the energy maps of the even-even nuclei with $N=158-168$ are shown.
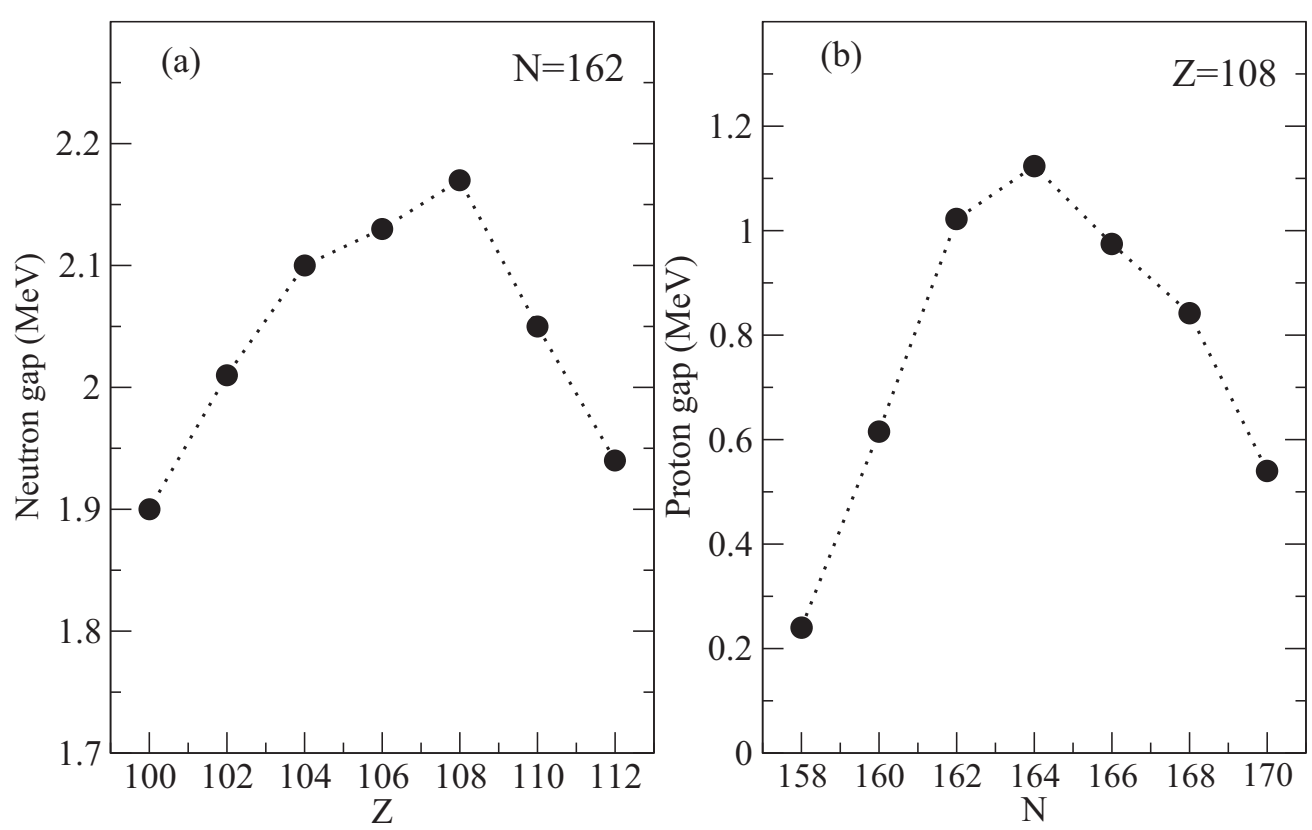

FIG. 4. The deformed neutron shell gap of $N=162$ isotones as a function of proton number (left panel), and the proton shell gap of Hs isotopes as a function of the number of neutrons (right panel). 

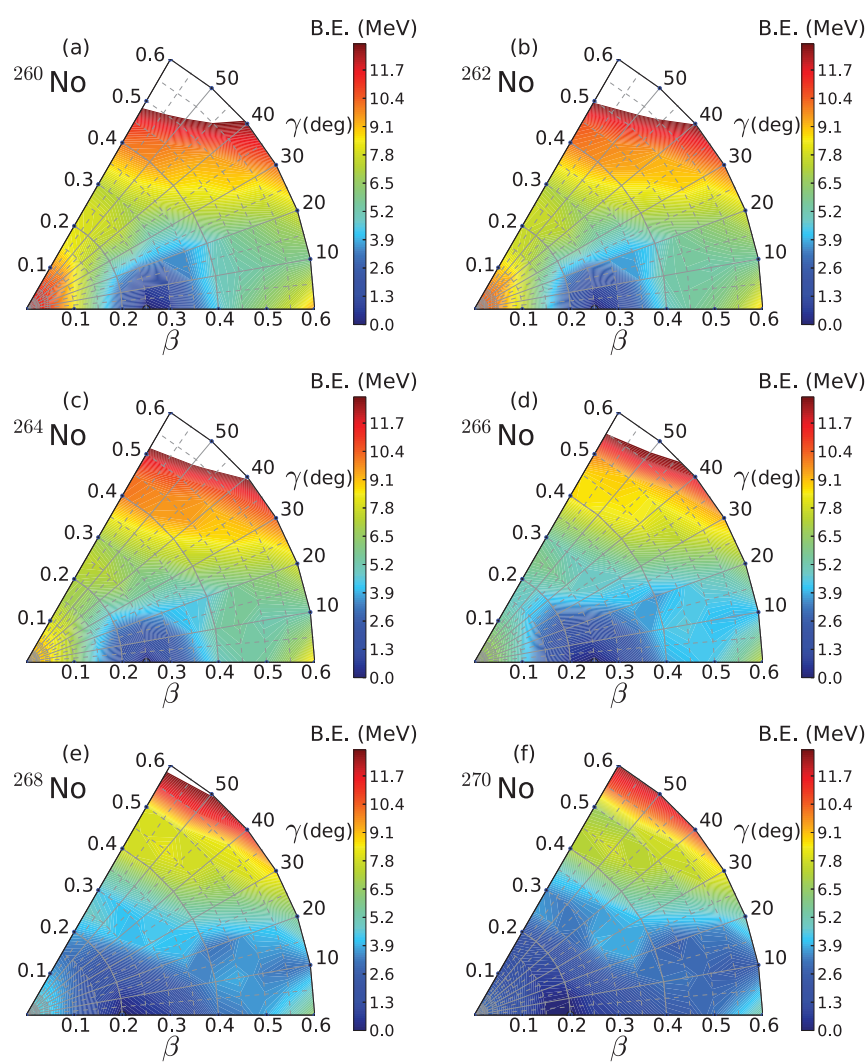

FIG. 5. (Color online) Self-consistent RHB triaxial energy maps of even-even No isotopes in the $\beta-\gamma$ plane $\left(0^{\circ} \leqslant \gamma \leqslant 60^{\circ}\right)$. For each nucleus energies are normalized with respect to the binding energy of the absolute minimum.

Even though we consider five chains of isotopes with $Z$ ranging from 102 to 110 , the evolution of the energy maps with neutron number is remarkably uniform. For each element the three lightest isotopes with $N=158,160,162$ are characterized by well-developed, deep mean-field minima at prolate deformations $\beta \approx 0.25$. Starting from $N=164$ the energy surfaces display a pronounced softness with respect to triaxial deformations. The region around equilibrium minima becomes extended both in the $\beta$ and $\gamma$ directions, and this effect is especially marked at $N=168$ for which the model predicts $\gamma$-soft energy surfaces that extend from the spherical configuration to $\beta \approx 0.3$, and even larger for $\mathrm{No}, \mathrm{Rf}$, and $\mathrm{Sg}$.

Heavier isotopes eventually become spherical but for the present analysis and future experimental studies especially interesting are nuclei close to $N=162$. To model lowenergy excitation spectra, we start from the self-consistent microscopic energy surfaces shown in Figs. 5-9. The neutron and proton single-quasiparticle energies and wave functions, generated by constrained self-consistent RHB calculations, provide the microscopic input for the parameters of the quadrupole collective Hamiltonian at each point on the energy surface: the mass parameters, the moments of inertia, and the collective potential, as functions of the deformations $\beta$ and $\gamma$. In the simplest approximation the moments of inertia are calculated using the Inglis-Belyaev formula $[38,39]$, the mass parameters associated with the two quadrupole collective coordinates are computed in the cranking approximation
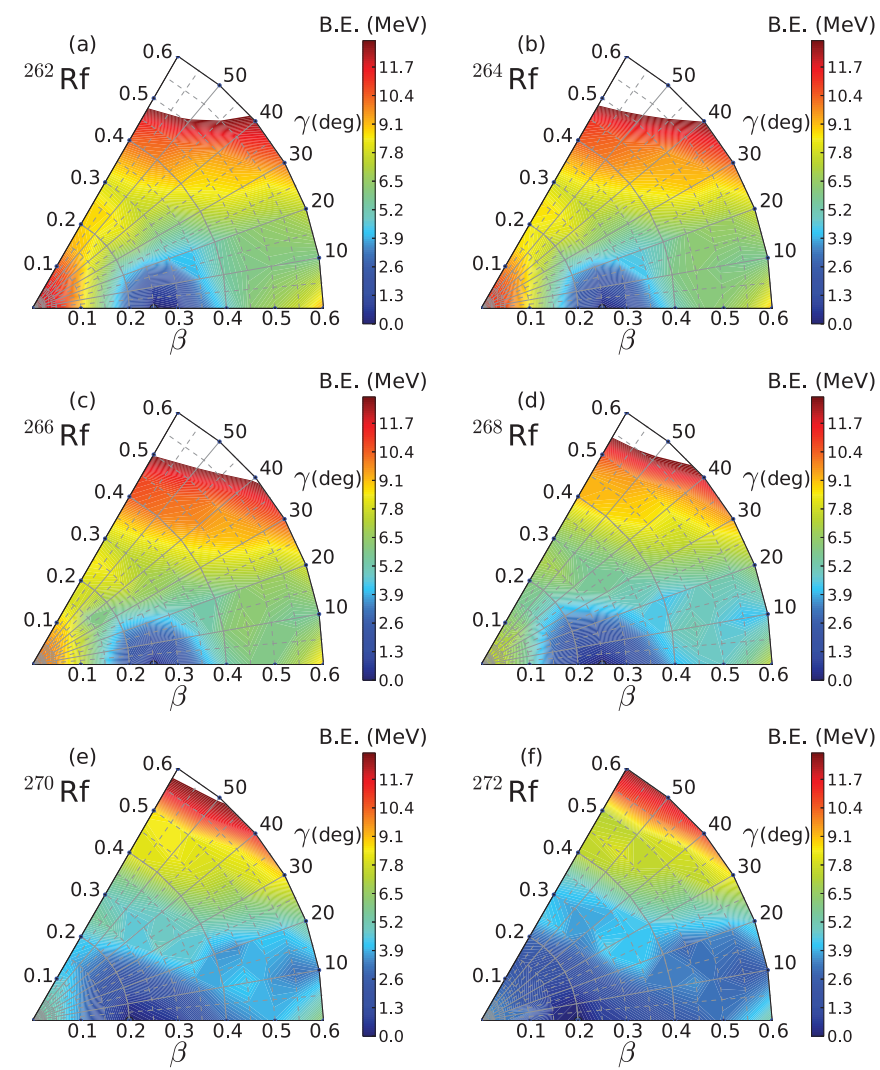

FIG. 6. (Color online) Same as described in the caption to Fig. 5 but for the isotopic chain of Rf.

[40], and the collective potential is obtained by subtracting the energy of the zero-point motion from the total-energy surface [40]. The diagonalization of the Hamiltonian yields the excitation spectra and collective wave functions that are used in the calculation of various observables, e.g., electromagnetic transition probabilities $[23,33]$.

As an example, in Fig. 10 we display the low-energy collective spectrum of ${ }^{256} \mathrm{Rf}$, in comparison to very recent data on the yrast sequence of this nucleus [18]. In addition to the yrast ground-state band, in deformed and transitional nuclei excited states are also assigned to (quasi-) $\beta$ and $\gamma$ bands. This is done according to the distribution of the projection $K$ of the angular momentum $I$ on the $z$ axis of the body-fixed frame. Excited states with predominant $K=2$ components in the wave function are assigned to the $\gamma$ band, whereas the $\beta$ band comprises the states above the yrast characterized by dominant $K=0$ components. The calculated excitation energies have been rescaled to reproduce the adopted energy of the state $2_{1}^{+}: 44 \mathrm{keV}$ [18]. This scaling is necessary because of the well-known fact that the Inglis-Belyaev (IB) moments of inertia and cranking mass parameters are actually smaller than the corresponding empirical values, due to the omission of time-odd components of the mean-field (the so-called Thouless-Valatin dynamical rearrangement contributions).

The resulting excitation spectrum of the collective Hamiltonian, based on the functional DD-PC1, is in very good agreement with available data for the ground-state band of ${ }^{256} \mathrm{Rf}$. 

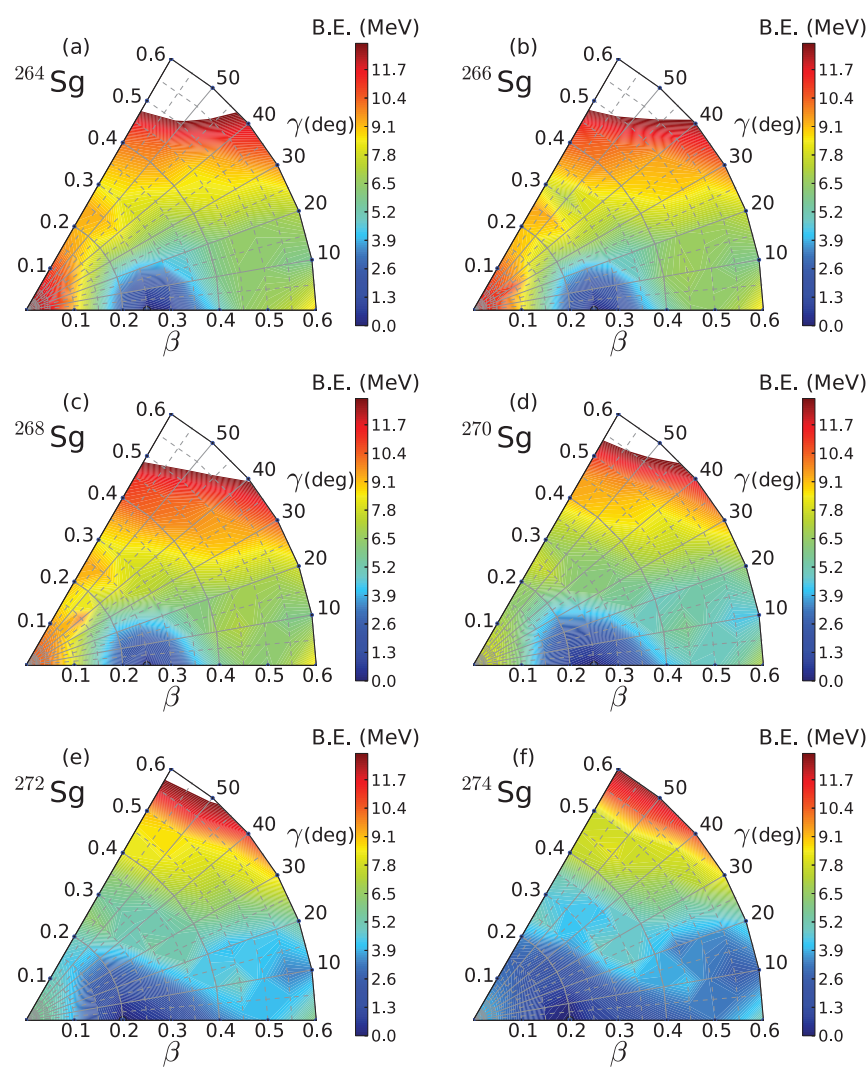

FIG. 7. (Color online) Same as described in the caption to Fig. 5 but for the isotopic chain of $\mathrm{Sg}$.

The scaling of excitation energies by a common factor does not affect the wave functions and, therefore, the calculation of transition probabilities. Fig. 10 includes the calculated $B(E 2)$ values, in Weisskopf units (W.u.), for the transitions in the ground-state band, and from the band heads of the $\beta$ and $\gamma$ bands to the ground-state band. It should be emphasized that besides the single scaling factor determined by the empirical excitation energy of the state $2_{1}^{+}$, the calculation is completely parameter free, i.e., by using a structure model based on self-consistent mean-field single-particle solutions, physical observables, such as transition probabilities and spectroscopic quadrupole moments, are calculated in the full configuration space and there is no need for effective charges. Using the bare value of the proton charge in the electric quadrupole operator $\hat{\mathcal{M}}(E 2)$, transition probabilities between eigenstates of the collective Hamiltonian can be directly compared to data.

In Figs. 11-14 we analyze the isotopic dependence of several observables of low-energy spectra, calculated using the collective Hamiltonian based on the functional DD-PC1, that characterize the transitions between axially symmetric rotors, $\gamma$-soft rotors, and, eventually, spherical vibrators. One of the distinct characteristics of shape transitions is the evolution of the ratio $R_{4 / 2}$ of excitation energies of the yrast states $4_{1}^{+}$ and $2_{1}^{+}$. For an axially symmetric rotor $R_{4 / 2}=3.33$, values between 2.5 and 2 are typical for a nucleus characterized by a $\gamma$-unstable potential [41] and, finally, $R_{4 / 2}=2.0$ for a spherical vibrator. In Fig. 11 we plot the theoretical values of $R_{4 / 2}$ for No, Rf, Sg, Hs, and Ds, as functions of the neutron
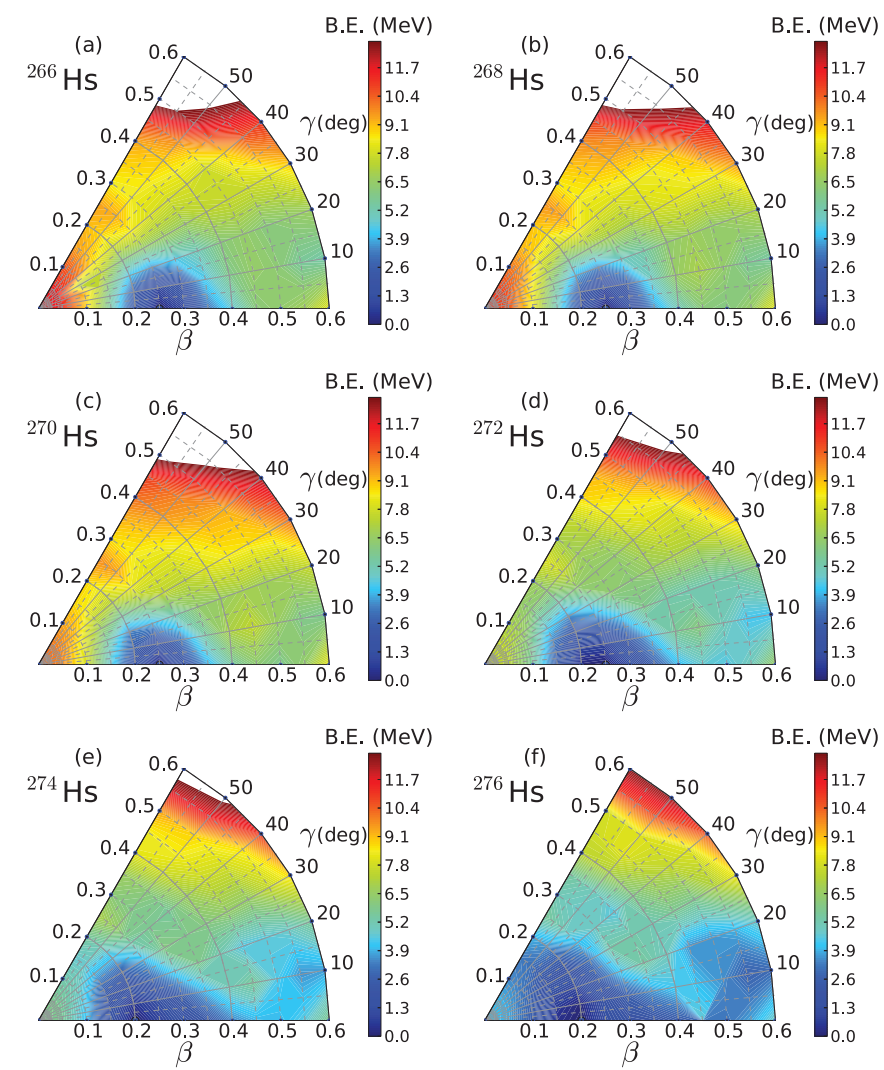

FIG. 8. (Color online) Same as described in the caption to Fig. 5 but for the isotopic chain of Hs.

number. A remarkable result is that all five isotopic chains display minimal variation of $R_{4 / 2}$ from the axial rigid rotor value 3.33 in the interval $N=158-166$. For neutron numbers $N \geqslant 168$ the values of $R_{4 / 2}$ start decreasing, reflecting the developing $\gamma$ softness of the potentials (cf. Figs. 5-9). We notice a regularity with respect to the proton number: the effect is more pronounced for neutron-rich nuclei, that is, $R_{4 / 2} \approx 2.6$ in No already at $N=170$, whereas for this neutron number the ratio only decreases to $\approx 3.2$ in Ds.

The development of softness in the collective potential of lighter neutron-rich transactinides, as shown by the energy surfaces in the $\beta-\gamma$ plane, is also reflected in another characteristic observable: the ratio of reduced transition probabilities $R=B\left(E 2 ; 4_{1}^{+} \rightarrow 2_{1}^{+}\right) / B\left(E 2 ; 2_{1}^{+} \rightarrow 0_{1}^{+}\right)$. Figure 12 displays this ratio as a function of neutron number for the five isotopic chains of No, Rf, $\mathrm{Sg}, \mathrm{Hs}$, and Ds. The minimal value $R=1.43$ characterizes the rigid-rotor limit and the $\gamma$-independent $\beta$-rigid Wilets-Jean limit [41] of the collective model. The ratio increases for smaller and/or softer axial deformations, reaching the value $R=2$ in the spherical vibrator limit. One notices that also for this observable the dependence on neutron number is very weak in the interval $N=158-166$, and the calculated values of $R$ are close to the rotational limit for all five isotopic chains. For more neutron-rich nuclei $R$ displays a rather steep increase, especially for No and Rf, but it would only reach the vibrational limit for much larger number of neutrons.

Another interesting feature is the departure from the rotational limit as a function of angular momentum for yrast states, 

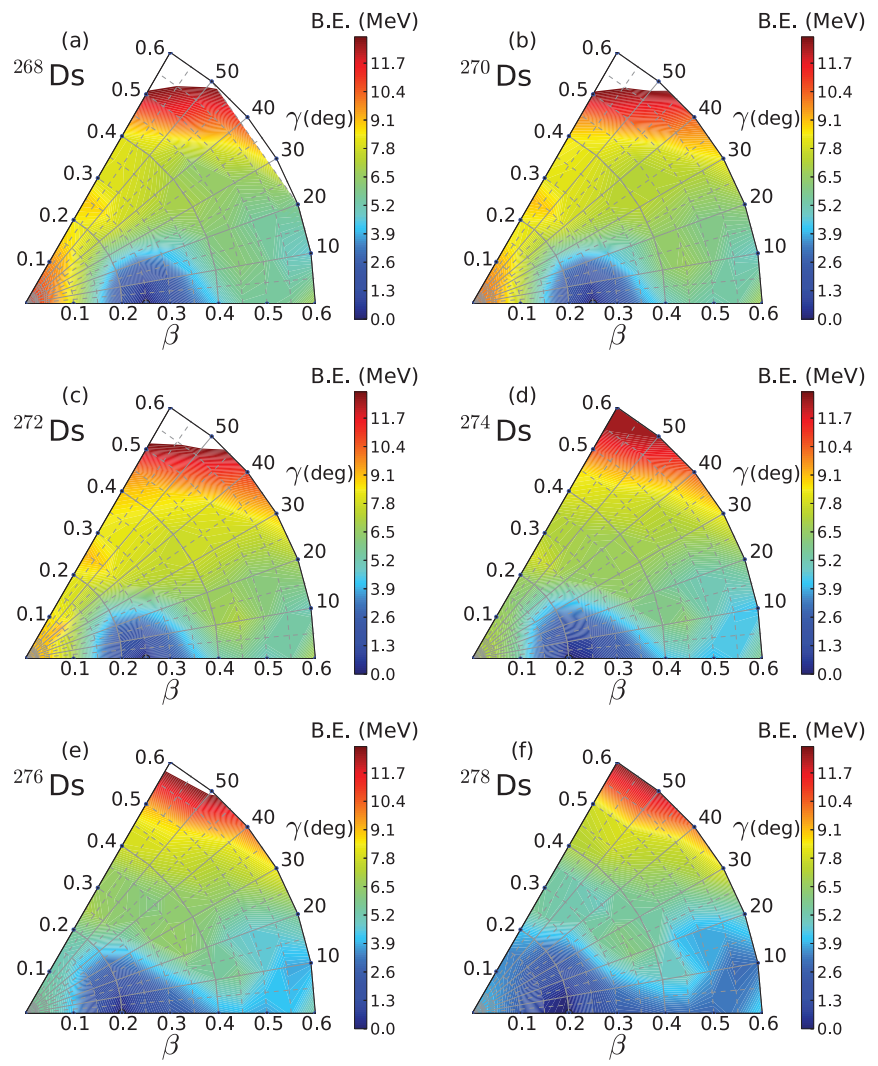

FIG. 9. (Color online) Same as described in the caption to Fig. 5 but for the isotopic chain of Ds.

as characterized by the $B(E 2)$ values for transitions within the ground-state band. In Fig. 13 we plot the $B(E 2)$ values for ground-state band transitions, normalized to $B\left(E 2 ; 2_{1}^{+} \rightarrow\right.$ $0_{1}^{+}$), in the $N=158,160,162,164,166$, and 168 isotones of No, Rf, Sg, Hs, and Ds. The dash-dotted and dashed lines

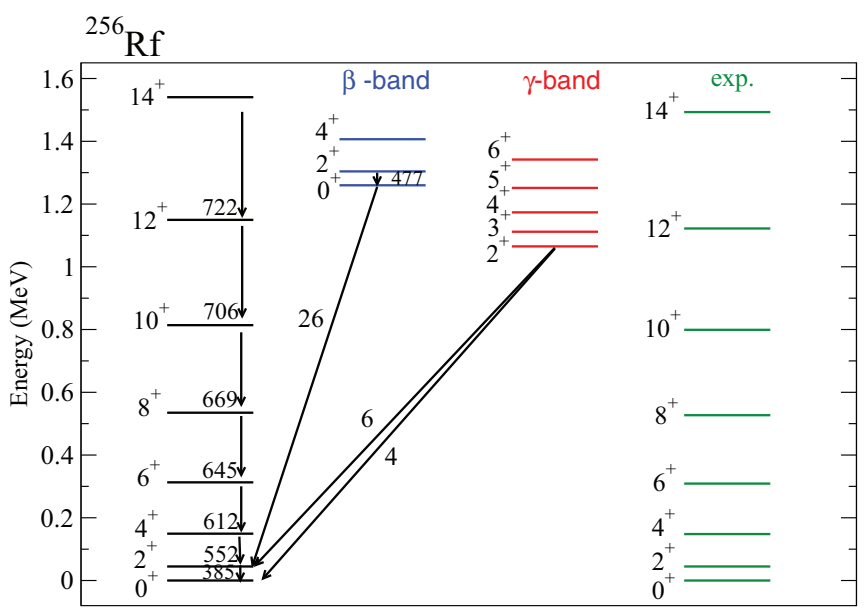

FIG. 10. (Color online) The low-energy collective spectrum of ${ }^{256} \mathrm{Rf}$ calculated with the DD-PC1 relativistic density functional compared to the experimental yrast sequence [18].

denote the rotational and vibrational limits of the collective model, respectively. Remarkably, all five isotopic chains display $B(E 2)$ values that almost exactly coincide with the rotational limit up to and above angular momentum $14^{+}$, for neutron number $N \leqslant 164$. Only starting from $N=166$ one notices a slight deviation from the rotational limit, increasing with angular momentum.

The evolution of $\gamma$ softness, that is, the deviation from the axial rigid-rotor limit, leads to the mixing between bands with different values of the angular-momentum projection $K$ quantum number, e.g., between the $K=0$ bands and the $\gamma$ band $(K=2)$. The level of $K$-mixing is reflected in the staggering in the energy between odd- and even-spin states in the (quasi-) $\gamma$ bands. The staggering can be quantified by considering the differential quantity [42]

$$
S(J)=\frac{\left\{E\left[J_{\gamma}^{+}\right]-E\left[(J-1)_{\gamma}^{+}\right]\right\}-\left\{E\left[(J-1)_{\gamma}^{+}\right]-E\left[(J-2)_{\gamma}^{+}\right]\right\}}{E\left[2_{1}^{+}\right]},
$$

which measures the displacement of the $(J-1)_{\gamma}^{+}$level relative to the average of its neighbors, $J_{\gamma}^{+}$and $(J-2)_{\gamma}^{+}$, normalized to the energy of the first excited state of the ground state band $2_{1}^{+}$. Because of its differential form, $S(J)$ is very sensitive to structural changes. For an axially symmetric rotor $S(J)$ is constant. In a nucleus with deformed $\gamma$-soft potential it oscillates between negative values for even-spin states and positive values for odd-spin states, with the magnitude slowly increasing with spin. For a triaxial potential the level clustering in the (quasi-) $\gamma$-band is opposite and $S(J)$ oscillates between positive values for even-spin states and negative values for odd-spin states. In this case the magnitude of $S(J)$ increases more rapidly with spin, as compared with the $\gamma$-soft potential. Figure 14 shows the quantity $S(J)$ in the $\gamma$ bands of No, Rf, $\mathrm{Sg}$, Hs, and Ds with $N=158-168$, as a function of angular momentum. Again, $S(J)$ displays an almost constant value up to relatively high angular momentum for all isotopic chains with neutron number $N \leqslant 164$. A signature of staggering in the $\gamma$ band is observed starting from $N=166$, at first only in No and Rf, and a consistent staggering of $S(J)$ is predicted for $N=168$. For No and Rf, in particular, $S(J)$ changes sign between even- and odd-spin states: negative for even spins and positive for odd spins, consistent with the $\gamma$-soft energy surfaces of these isotopes.

Finally, we briefly discuss an interesting possibility for the occurrence of shape-phase transitions and critical-point phenomena in the region of heavy and superheavy nuclei. The transition between different shapes in most isotopic or isotonic sequences is gradual but in some cases the addition/subtraction of only a few nucleons leads to rather rapid changes in equilibrium shapes. Ground-state phase transitions in nuclei correspond to first- and second-order quantum phase transitions (QPTs) between competing ground-state phases induced by variation of a nonthermal control parameter (number of 


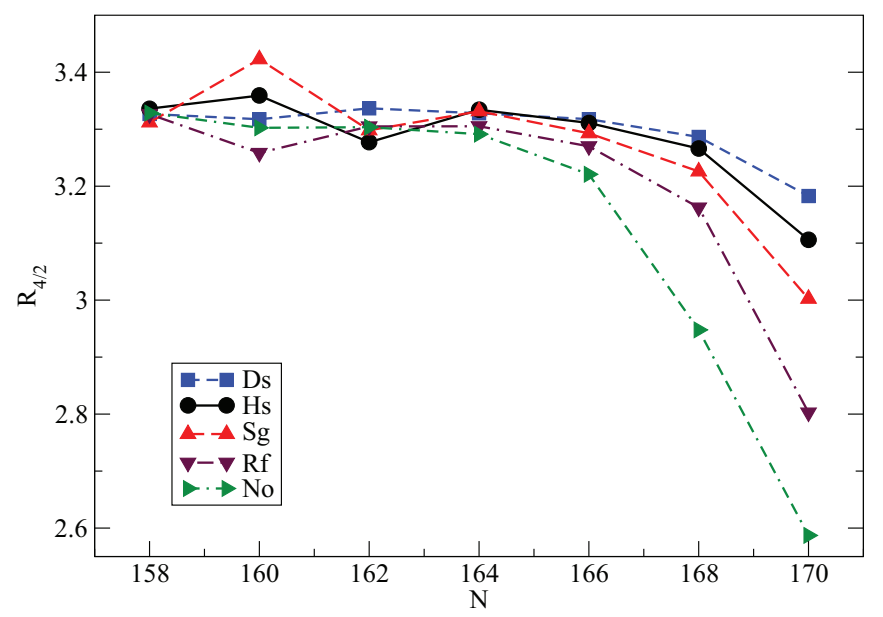

FIG. 11. (Color online) The ratio $R_{4 / 2}$ of excitation energies of the yrast states $4_{1}^{+}$and $2_{1}^{+}$as a function of the neutron number for the isotopic chains of No, Rf, $\mathrm{Sg}$, Hs, and Ds.

nucleons) at zero temperature. During the last decade nuclear QPTs have been investigated extensively, both in experimental studies and employing a variety of theoretical models (cf. Ref. [43] for a recent review). A list of problems that have not been completely solved includes the identification of observables that can be related to order parameters, the degree to which discontinuities at a phase transitional point are smoothed out in finite nuclei, and the question of how precisely can a point of phase transition be associated with a particular isotope, considering that the physical control parameter, i.e., nucleon number, is not continuous but takes only discrete integer values.

Since in systems with a finite number of particles phase transitions are actually smoothed out, one expects that signatures of abrupt changes of structure properties would be observed more clearly in heavy nuclei. In very heavy systems, however, spectroscopic data are often limited to yrast sequences and there is not enough information on observables that can

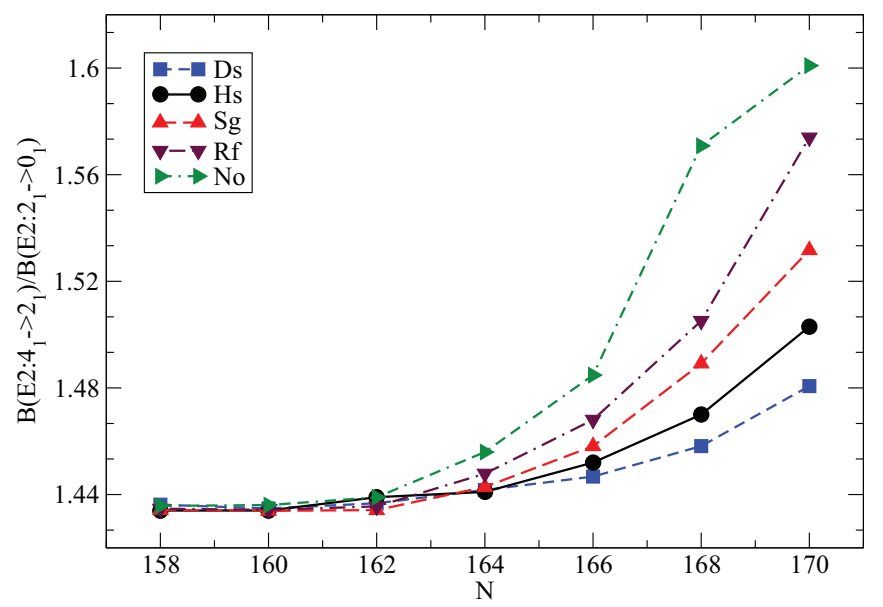

FIG. 12. (Color online) The ratio of reduced transition probabilities $B\left(E 2 ; 4_{1}^{+} \rightarrow 2_{1}^{+}\right) / B\left(E 2 ; 2_{1}^{+} \rightarrow 0_{1}^{+}\right)$as a function of the neutron number for the isotopic chains of No, $\mathrm{Rf}, \mathrm{Sg}, \mathrm{Hs}$, and Ds.

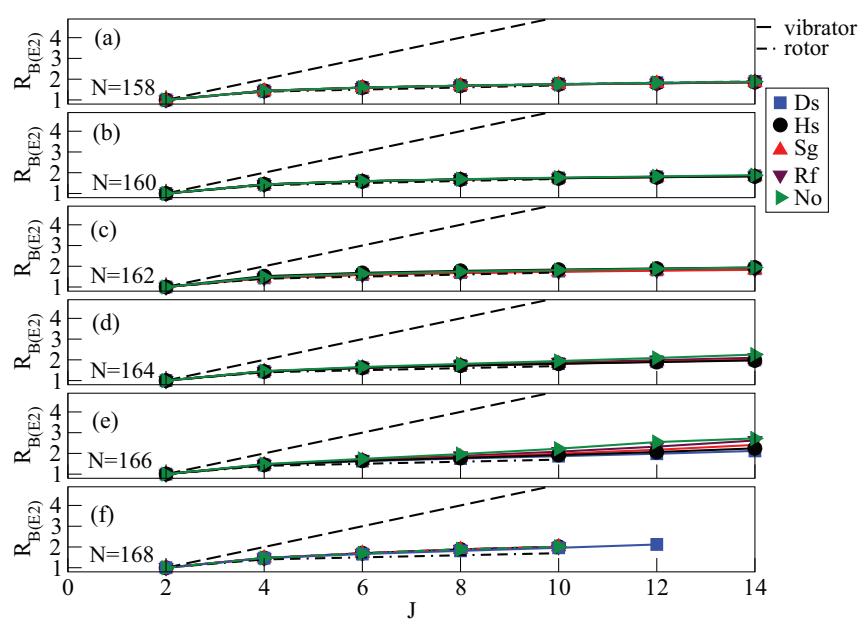

FIG. 13. (Color online) $B(E 2)$ values for transition between yrast states, normalized to $B\left(E 2 ; 2_{1}^{+} \rightarrow 0_{1}^{+}\right)$, in the $N=$ $158,160,162,164,166$, and 168 isotones of No, Rf, $\mathrm{Sg}, \mathrm{Hs}$, and Ds.

be related to quantum order parameters. Nevertheless, it is interesting to investigate such transitions theoretically. The evolution of shapes in the No isotopic chain, in particular, shows an interesting feature close to neutron number $N=168$ (cf. Fig. 5). The deformation energy surface displays a rather flat prolate minimum that extends in the axial deformation parameter, and a parabolic dependence on $\gamma$ in the region of the minimum. The flat bottom of the potential has been considered a signature of possible phase transition because it allows for fluctuations of collective variables. The phase transition between axially deformed and spherical nuclei involves two degrees of freedom-the deformations $\beta$ and $\gamma$. The critical point of this phase transition, denoted $X(5)$ [44], corresponds to the particular case in which the $\beta$ and $\gamma$ degrees of freedom are decoupled, and an approximate analytic solution at the critical point of phase transition can be expressed in terms of zeros of Bessel functions of irrational order. Evidence for the empirical realization of $\mathrm{X}(5)$ phase transition was first

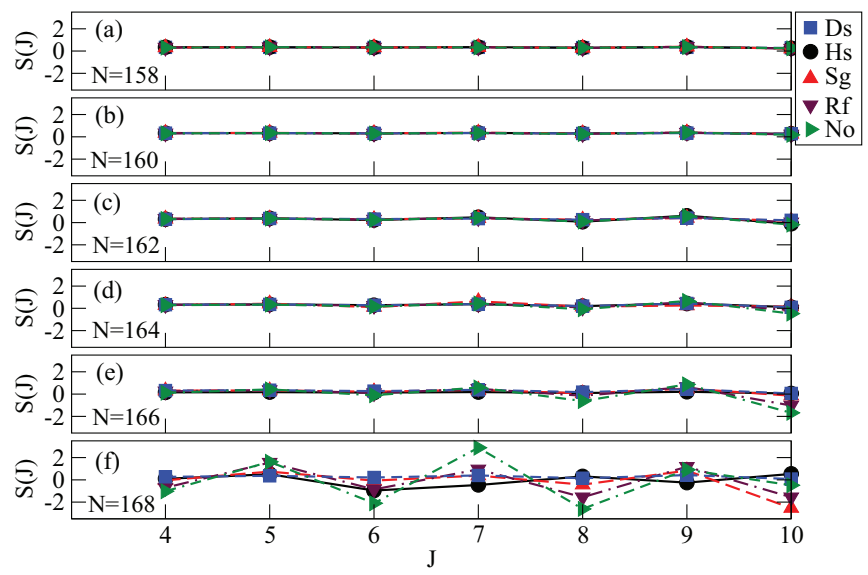

FIG. 14. (Color online) Staggering $S(J)$ [Eq. (4)] in the $\gamma$ bands of No, Rf, Sg, Hs, and Ds with $N=158-168$. 


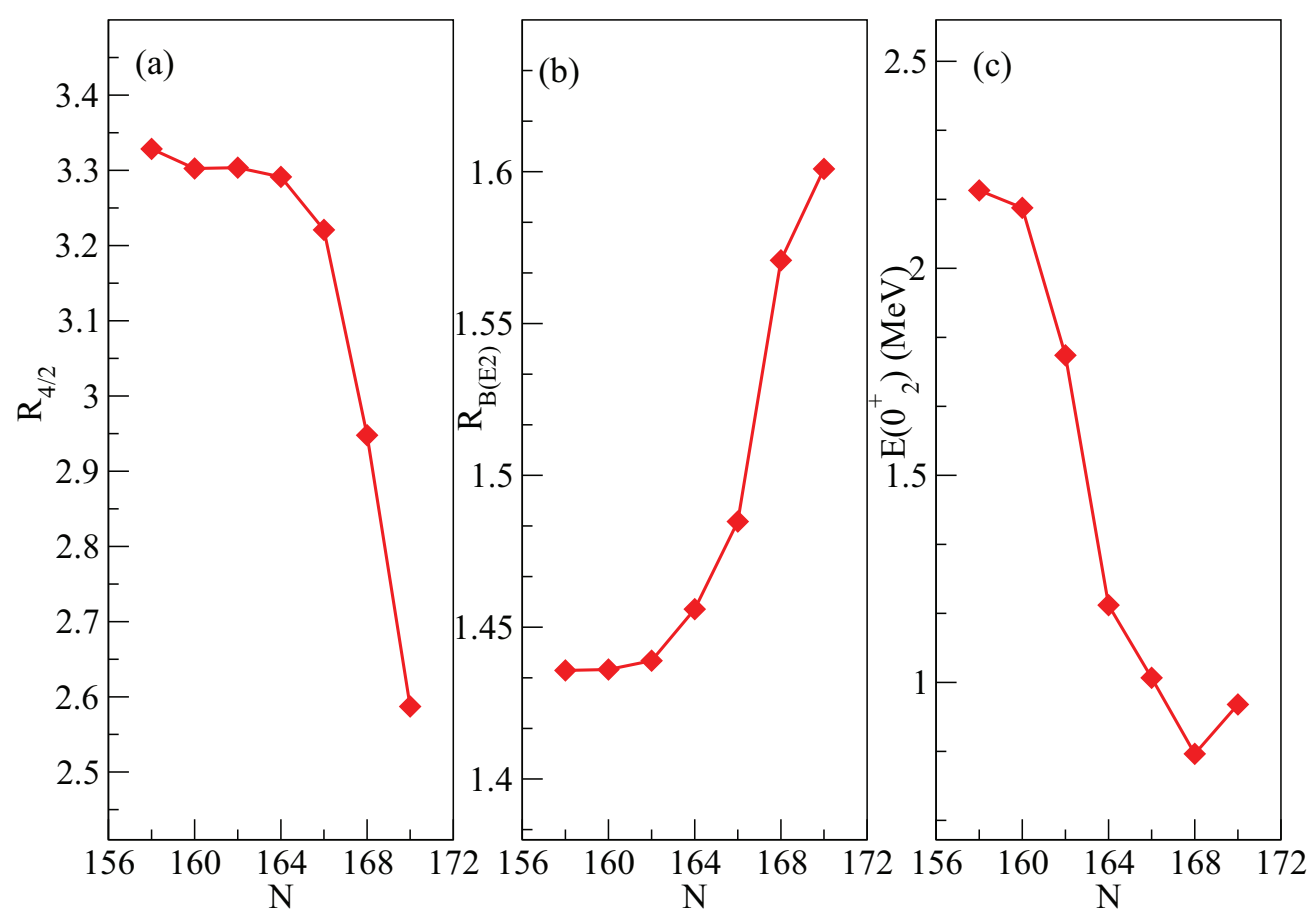

FIG. 15. (Color online) The ratio $R_{4 / 2}$ of excitation energies of the levels $4_{1}^{+}$and $2_{1}^{+}$(a), the ratio of reduced transition probabilities $R=B\left(E 2 ; 4_{1}^{+} \rightarrow 2_{1}^{+}\right) / B\left(E 2 ; 2_{1}^{+} \rightarrow 0_{1}^{+}\right)(\mathrm{b})$, and the excitation energy of the level $0_{2}^{+}$(c), in No isotopes as functions of the number of neutrons.

reported for ${ }^{152} \mathrm{Sm}$ and other $N=90$ isotones [45]. In a series of studies [46-48] we have analyzed microscopic signatures of nuclear ground-state shape phase transitions in $\mathrm{Nd}$ isotopes using excitation spectra and collective wave functions obtained by diagonalization of a five-dimensional Hamiltonian for quadrupole vibrational and rotational degrees of freedom, with parameters determined by constrained self-consistent relativistic mean-field calculations for triaxial shapes. It has been shown that a number of observables, e.g., energy gaps between the ground state and the excited vibrational states with zero angular momentum, isomer shifts, and monopole transition strengths, exhibit sharp discontinuities at neutron number $N=90$, characteristic of a first-order quantum phase transition.

For the sequence of No nuclei, in Fig. 15 we display the isotopic dependence of three characteristic quantities, calculated with the collective Hamiltonian based on the DDPC1 functional: the ratio $R_{4 / 2}$ of excitation energies of the yrast states $4_{1}^{+}$and $2_{1}^{+}$, the ratio of reduced transition probabilities $R=B\left(E 2 ; 4_{1}^{+} \rightarrow 2_{1}^{+}\right) / B\left(E 2 ; 2_{1}^{+} \rightarrow 0_{1}^{+}\right)$, and the excitation energy of the first-excited (second) $0^{+}$state. It is interesting to note that all three observables display a pronounced variation between $N=166$ and $N=170$. For $N=168$, in particular, we can compare the calculated values with the predictions of the $\mathrm{X}(5)$ analytical model for the critical point of shape phase transition [44]: $R_{4 / 2}=2.94$ for ${ }^{270}$ No compared to 2.91 in the $\mathrm{X}(5)$ model, and the calculated ratio of reduced transition probabilities is $R=1.57$ for ${ }^{270}$ No while the $\mathrm{X}(5)$ value is 1.58 . This is indeed a remarkable agreement between the microscopic calculation based on a global energy density functional and parameter-free $\mathrm{X}(5)$ predictions for spectroscopic properties at the point of phase transition. In addition, the pronounced dip in the excitation energy of the band-head $0_{2}^{+}$of the quasi- $\beta$ band can be attributed to the softness of the potential with respect to $\beta$ deformation. This example illustrates the richness of structure phenomena that one can expect to find in very heavy nuclei with $Z \geqslant 100$, including the possible occurrence of shape phase transitions. We emphasize, however, that the physical control parameterthe nucleon number-is not continuous and thus in general a microscopic calculation cannot exactly reproduce the point of phase transition.

\section{SUMMARY AND OUTLOOK}

The framework of relativistic nuclear energy density functionals (EDFs) has been applied to a study of the structure of transactinide nuclei. Based on the functional DD-PC1, that has successfully been tested in various mass regions including superheavy nuclei, and on a separable pairing interaction, self-consistent relativistic Hartree-Bogoliubov calculations have been performed for several isotopic chains from Fm to $\mathrm{Fl}$, with neutron number in the interval $154 \leqslant N \leqslant 172$. Assuming axially symmetric shapes, in particular, we have analyzed the deformed shell gap for neutrons at $N=162$ and how it affects the stability of nuclei around ${ }^{270} \mathrm{Hs}$. Triaxial deformation energy surfaces have been computed for the even-even isotopes of No, Rf, $\mathrm{Sg}, \mathrm{Hs}$, and Ds, with $N=158-168$. Although $Z$ ranges from 102 to 110 , all the isotones with $N=158,160,162$ display similar deformation energy maps: well-developed, deep mean-field minima at 
prolate deformations $\beta \approx 0.25$. From $N=164$ on, a marked softness with respect to triaxial deformations develops, and this effect is especially visible at $N=168$ for which the model predicts $\gamma$-soft energy surfaces that extend from the spherical configuration to large axial deformations.

A quadrupole collective Hamiltonian, with parameters determined by self-consistent constrained triaxial RHB calculations, has been used to calculate low-energy spectra of these isotopic chains. The isotopic dependence of several observables that characterize the transition between axially symmetric rotors and $\gamma$-soft rotors has been examined: the ratio $R_{4 / 2}$ of excitation energies of the yrast states $4_{1}^{+}$and $2_{1}^{+}$, the ratio of reduced transition probabilities $R=B\left(E 2 ; 4_{1}^{+} \rightarrow\right.$ $\left.2_{1}^{+}\right) / B\left(E 2 ; 2_{1}^{+} \rightarrow 0_{1}^{+}\right), B(E 2)$ values for transitions within the ground-state band, and the level of $K$-mixing as reflected in the staggering in the energy between odd- and even-spin states in the (quasi-) $\gamma$ bands. We have also analyzed an interesting example of a possible $X(5)$ shape-phase transition in the sequence of No isotopes.
The detailed predictions for spectroscopic properties of transactinide nuclei, reported in this work, open a new perspective for future theoretical studies in this mass region. The self-consistent mean-field approach and "beyond meanfield" models that take into account collective correlations, will be applied to the analysis of high- $K$ isomeric states [49], calculation of triaxial and octupole fission barriers [50], and, in particular, the evolution of deformed shell structuresa common issue for all self-consistent mean-field models [15].

\section{ACKNOWLEDGMENTS}

The authors are grateful to Dieter Ackermann, Shan-Gui Zhou, and Bing-Nan Lu for valuable discussions. This work has been supported in part by the Finland Distinguished Professor Programme (FiDiPro), and by the MZOS - Project No. 1191005-1010.
[1] S. Hofmann and G. Münzenberg, Rev. Mod. Phys. 72, 733 (2000).

[2] Yu. Ts. Oganessian, J. Phys. G 34, R165 (2007).

[3] S. Hofmann, Radiochim. Acta 99, 405 (2011).

[4] K. Morita et al., J. Phys. Soc. Jpn. 81, 103201 (2012).

[5] Zhang Zhi-Yuan et al., Chin. Phys. Lett. 29, 012502 (2012).

[6] P.-H. Heenen and W. Nazarewicz, Europhys. News 33, 1 (2009).

[7] M. Leino and F. P. Heßberger, Annu. Rev. Nucl. Part. Sci. 54, 175 (2004).

[8] R.-D. Herzberg and P. T. Greenlees, Prog. Part. Nucl. Phys. 61, 674 (2008)

[9] R.-D. Herzberg and D. M. Cox, Radiocim. Acta 99, 441 (2011).

[10] P. Möller and J. R. Nix, J. Phys. G: Nucl. Part. Phys. 20, 1681 (1994).

[11] P. Möller, J. R. Nix, W. D. Myers, and W. J. Swiatecki, At. Data Nucl. Data Tables 59, 185 (1995).

[12] A. Sobiczewski, Fiz. Elem. Chastits At. Yadra 25, 295 (1994) [Phys. Part. Nucl. 25, 119 (1994)].

[13] A. Sobiczewski and K. Pomorski, Prog. Part. Nucl. Phys. 58, 292 (2007).

[14] M. Bender, P.-H. Heenen, and P.-G. Reinhard, Rev. Mod. Phys. 75, 121 (2003).

[15] M. Bender and P.-H. Heenen, arXiv:1210.2780

[16] Extended Density Functionals in Nuclear Structure Physics, Lecture Notes in Physics 641, edited by G. A. Lalazissis, P. Ring, and D. Vretenar (Springer, Heidelberg, 2004).

[17] Bing-Nan Lu, Jie Zhao, En-Guang Zhao, and Shan-Gui Zhou, arXiv:1304.6830.

[18] P. T. Greenlees et al., Phys. Rev. Lett. 109, 012501 (2012).

[19] Zhen-Hua Zhang, Jie Meng, En-Guang Zhao, and Shan-Gui Zhou, Phys. Rev. C 87, 054308 (2013).

[20] A. V. Afanasjev and O. Abdurazakov, Phys. Rev. C 88, 014320 (2013).

[21] D. Vretenar, A. V. Afanasjev, G. A. Lalazissis, and P. Ring, Phys. Rep. 409, 101 (2005).

[22] J. Meng, H. Toki, S. G. Zhou, S. Q. Zhang, W. H. Long, and L. S. Geng, Prog. Part. Nucl. Phys. 57, 470 (2006).
[23] T. Nikšić, D. Vretenar, and P. Ring, Prog. Part. Nucl. Phys. 66, 519 (2011).

[24] T. Nikšić, D. Vretenar, and P. Ring, Phys. Rev. C 78, 034318 (2008).

[25] Z. P. Li, J. M. Yao, D. Vretenar, T. Nikšić, H. Chen, and J. Meng, Phys. Rev. C 84, 054304 (2011).

[26] Z. P. Li, T. Nikšić, D. Vretenar, and J. Meng, Phys. Rev. C 81, 034316 (2010).

[27] H. Abusara, A. V. Afanasjev, and P. Ring, Phys. Rev. C 85, 024314 (2012).

[28] V. Prassa, T. Nikšić, G. A. Lalazissis, and D. Vretenar, Phys. Rev. C 86, 024317 (2012).

[29] Y. Tian, Z. Y. Ma, and P. Ring, Phys. Lett. B 676, 44 (2009).

[30] T. Nikšić, P. Ring, D. Vretenar, Y. Tian, and Z. Y. Ma, Phys. Rev. C 81, 054318 (2010).

[31] J. F. Berger, M. Girod, and D. Gogny, Comp. Phys. Comm. 63, 365 (1991).

[32] Y. K. Gambhir, P. Ring, and A. Thimet, Ann. Phys. (NY) 198, 132 (1990).

[33] T. Nikšić, Z. P. Li, D. Vretenar, L. Próchniak, J. Meng, and P. Ring, Phys. Rev. C 79, 034303 (2009).

[34] Z. Patyk and A. Sobiczewski, Nucl. Phys. A 533, 132 (1991).

[35] A. Sobiczewski, I. Muntian, and Z. Patyk, Phys. Rev. C 63, 034306 (2001).

[36] M. Warda and J. L. Egido, Phys. Rev. C 86, 014322 (2012).

[37] M. Wang, G. Audi, A. H. Wapstra, F. G. Kondev, M. MacCormick, X. Xu, and B. Pfeiffer, Chin. Phys. C 36, 1603 (2012).

[38] D. R. Inglis, Phys. Rev. 103, 1786 (1956).

[39] S. T. Belyaev, Nucl. Phys. 24, 322 (1961).

[40] M. Girod and B. Grammaticos, Nucl. Phys. A 330, 40 (1979).

[41] L. Wilets and M. Jean, Phys. Rev. 102, 788 (1956).

[42] N. Zamfir and R. F. Casten, Phys. Lett. B 260, 265 (1991).

[43] P. Cejnar, J. Jolie, and R. F. Casten, Rev. Mod. Phys. 82, 2155 (2010).

[44] F. Iachello, Phys. Rev. Lett. 87, 052502 (2001). 
[45] R. F. Casten and N. V. Zamfir, Phys. Rev. Lett. 87, 052503 (2001).

[46] T. Nikšić, D. Vretenar, G. A. Lalazissis, and P. Ring, Phys. Rev. Lett. 99, 092502 (2007).

[47] Z. P. Li, T. Nikšić, D. Vretenar, J. Meng, G. A. Lalazissis, and P. Ring, Phys. Rev. C 79, 054301 (2009).
[48] Z. P. Li, T. Nikšić, D. Vretenar, and J. Meng, Phys. Rev. C 80, 061301(R) (2009).

[49] D. Ackermann, Acta Phys. Pol. B 42, 577 (2011); Prog. Theo. Phys. Supp. 196, 255 (2012)

[50] Bing-Nan Lu, En-Guang Zhao, and Shan-Gui Zhou, Phys. Rev. C 85, 011301(R) (2012). 